\title{
Comportamento reológico de dispersões de argilas bentoníticas: efeitos do tipo de ferramenta, velocidade e tempo de agitação
}

\section{(Rheological behavior of suspensions of bentonite clays: effects of the type of tool, speed and stirring time)}

\author{
L. V. Amorim, C. M. Gomes, F. L. H. Silva ${ }^{1}$, H. C. Ferreira ${ }^{2}$ \\ ${ }^{1}$ Departamento de Eng. Química, ${ }^{2}$ Departamento de Eng. de Materiais \\ Universidade Federal da Paraíba \\ Av. Aprígio Veloso 882, Campina Grande, PB, 58109-970 \\ luciana@labdes.ufpb.br
}

\begin{abstract}
Resumo
Este trabalho teve como objetivo estudar a influência do tipo de ferramenta, da velocidade e do tempo de agitação nas propriedades reológicas de dispersões de quatro amostras de argilas bentoníticas sódicas industrializadas no Estado da Paraíba, Brasil. Foi realizado um planejamento fatorial do tipo $2^{3}$ para avaliar os fatores de entrada (tipo de ferramenta, velocidade e tempo de agitação) sobre as viscosidades aparente e plástica e o volume de filtrado. As dispersões foram preparadas na concentração de $4,86 \%$ em massa de argila $(24,3 \mathrm{~g}$ de argila em $500 \mathrm{~mL}$ de água deionizada) utilizando ferramentas do tipo palheta corrugada e em forma de borboleta, duas velocidades (10.000 rpm e $17.000 \mathrm{rpm}$ ) e dois tempos de agitação (10 e $20 \mathrm{~min}$ ). As viscosidades aparente e plástica foram determinadas em viscosímetro Fann 35A, e o volume de filtrado, em filtro prensa Fann. Através da regressão dos dados experimentais, verificou-se que as variáveis de entrada não apresentam influências estatisticamente significativas nas propriedades reológicas das dispersões estudadas, bem como, que dentre as quatro argilas estudadas apenas uma apresenta comportamento reológico de acordo com as normas vigentes para uso como agente tixotrópico para fluidos de perfuração à base de água doce.

Palavras-chave: planejamento experimental, bentonita, propriedades reológicas, fluidos de perfuração.
\end{abstract}

\begin{abstract}
This work had as objective to study the influence of the tool type, of the speed and of the stirring time in the rheological properties of suspensions of four samples of sodic bentonite clays supplied by industry from the state of Paraiba, Brazil. A factorial design of the type $2^{3}$ was developed to evaluate the entrance factors (tool type, speed and stirring time) in the apparent and plastic viscosities and fluid loss. The suspensions in the concentration of $4.86 \%(24.3 \mathrm{~g}$ of clay in 500 $m L$ of water) using tools of the type corrugated disk and butterfly form, two speeds (10,000 rpm and 17,000 rpm) and two stirring time $(10$ and $20 \mathrm{~min})$ were prepared. The apparent and plastic viscosities were measured in viscometer Fann 35A, and the fluid loss, in presses filter Fann. Through the regression of the experimental data, it was verified that the entrance variables don't present significant influences in the rheological properties of the studied dispersions, as well as, that just one presents rheological behavior in agreement with the effective norms for use as thixotropic agent for water based drilling fluids.
\end{abstract}

Keywords: factorial design, bentonite, rheological properties, drilling fluids.

\section{INTRODUÇÃO}

O Brasil possui o maior jazimento de argilas bentoníticas da América do Sul, localizado no Município de Boa Vista, Estado da Paraíba. Estas reservas são responsáveis por $96 \%$ da produção nacional de argila bruta e beneficiada (bentonita sódica) que são utilizadas para os mais diversos fins industriais, como areias de fundição, pelotização de minério de ferro, rejeito de dejetos animais, na indústria química e farmacêutica, clarificantes, captação de água e em fluidos de perfuração [1], aplicação de grande interesse na indústria de extração de petróleo.

Segundo o "American Petroleum Institute - API", fluidos de perfuração são definidos como fluidos de circulação usados em perfurações rotativas para desenvolver funções requeridas durante a operação de perfuração de poços [2]. Os fluidos de perfuração são tradicionalmente classificados de acordo com o seu constituinte principal em fluidos à base de gás, fluidos à 
base de óleo e fluidos à base de água. No início dos anos 90 foi desenvolvida uma nova classe de fluidos, denominada de fluidos sintéticos [3]. A utilização de um ou outro tipo de fluido depende da profundidade do poço e do tipo de formação a ser perfurada.

Os fluidos à base de água ativados com argila bentonítica são utilizados em todo o mundo [4] e, no Brasil, são amplamente empregados em perfurações de poços terrestres com até $1500 \mathrm{~m}$ de profundidade, sendo o consumo de bentonita em torno de 5200 t/ano. A bentonita é adicionada ao fluido para desempenhar uma ou várias das seguintes funções: aumentar a capacidade de limpeza do poço, reduzir as infiltrações nas formações permeáveis, formar uma membrana de baixa permeabilidade ("filter-cake"), promover a estabilidade do poço e evitar ou superar perda de circulação [5].

Segundo Souza Santos [6], as especificações e os métodos de ensaio estabelecidos pela Petrobras para a qualificação e aceitação de argilas bentoníticas para fluidos na exploração e produção de petróleo foram baseados nas normas romenas e do "American Petroleum Institute - API". No final dos anos 60, a Petrobras, a partir de dados práticos, modificou seus métodos de ensaio e suas especificações, sendo elaborada a norma EE78 [7]. Segundo esta normalização, as dispersões de argila eram preparadas na concentração de $6,0 \%$ em massa, com velocidade de agitação de $10.000 \mathrm{rpm}$. Os parâmetros reológicas avaliados são a viscosidade aparente, a viscosidade plástica e o filtrado API (volume de filtrado). Estas propriedades permitem avaliar a qualidade da argila, bem como, durante a operação de perfuração, conhecer as formações geológicas que estão sendo perfuradas e indicar tratamentos freqüentemente necessários ao fluido em virtude da incorporação de sólidos provenientes dos detritos gerados pela broca [8].

Atualmente, as especificações da Petrobras [9], classificam as argilas bentoníticas para uso como agente viscosificante em fluidos à base de água em: argila do tipo I e argila do tipo II. As argilas do tipo I são aquelas que em dispersão, na concentração de 4,86\% em massa, apresentam valores mínimos de viscosidades aparente e plástica de $15,0 \mathrm{cP}$ e 4,0 cP, respectivamente, e valor máximo de volume de filtrado de $18,0 \mathrm{~mL}$. As argilas do tipo II são aquelas que apresentam viscosidades mínimas aparente e plástica de 15,0 cP e 6,0 cP, respectivamente, e volume de filtrado máximo de 16,0 mL. O preparo das dispersões deve seguir padrões de velocidade e tempo de agitação, de forma a conduzir a argila a melhores condições de hidratação/inchamento, conferindo à dispersão as propriedades reológicas desejadas. A velocidade de agitação da dispersão deve estar compreendida entre 16.000 rpm e $20.000 \mathrm{rpm}$, enquanto que o tempo de agitação deve ser igual a 20 minutos. São recomendados agitadores da marca Hamilton Beach.

Em 1979, Assunção e Ferreira [10], estudaram a influência da concentração, velocidade e tempo de agitação nas propriedades reológicas de dispersões de argilas bentoníticas segundo métodos propostos pela norma EE-78 [7] e verificaram que o tempo e a velocidade de agitação influenciaram, respectivamente, a viscosidade aparente e o volume de filtrado.

Recentemente, Gomes e colaboradores [11], verificaram que o comportamento reológico de dispersões de argilas bentoníticas é bastante influenciado pelas condições de agitação utilizadas quando do seu preparo, sendo inadequado o uso de ferramentas de agitação do tipo rotor-estator.

Este trabalho teve como objetivo verificar a influência das variáveis tipo de ferramenta, velocidade e tempo de agitação no comportamento reológico de dispersões de quatro amostras de argilas bentoníticas sódicas industrializadas no Estado da Paraíba, Brasil.

\section{MATERIAISE MÉTODOS}

Foram estudadas quatro amostras de argilas bentoníticas industrializadas na forma sódica, cedidas por indústrias beneficiadoras de minérios e identificadas por Dolomil, Fungel, Brasgel e Brasgel PA. As argilas são provenientes das jazidas localizadas no Município de Boa Vista, Paraíba, Brasil e são compostas mineralogicamente por argilominerais do grupo da esmectita e com presença do mineral quartzo. A amostra Fungel apresenta ainda ilita e caulinita [12].

\section{Planejamento Experimental}

Para avaliar a influência das variáveis de entrada (tipo de ferramenta, velocidade e tempo de agitação) sobre o comportamento reológico de dispersões de argilas bentoníticas, foi utilizado um planejamento fatorial do tipo $2^{3}$, sendo os ensaios realizados em duplicata, totalizando 16 corridas experimentais para cada amostra [13]. A regressão dos dados

Tabela I - Níveis codificados e valores reais das variáveis de entrada do planejamento fatorial do tipo $2^{3}$.

[Table I - Coded factor levels and real values for factorial design $2^{3}$.]

\begin{tabular}{lcc}
\hline Variáveis de & \multicolumn{2}{c}{ Níveis codificados } \\
\cline { 2 - 3 } entrada & -1 & +1 \\
\cline { 2 - 3 } Ferramenta de agitação & $\mathrm{F} 1$ & $\mathrm{~F} 2$ \\
\hline Velocidade de agitação (rpm) & 10.000 & 17.000 \\
\hline Tempo de agitação (min) & 10 & 20 \\
\hline
\end{tabular}

Tabela II -Matriz de planejamento experimental.

[Table II - Factorial design matrix.]

\begin{tabular}{cccc}
\hline Experimentos & $\begin{array}{c}\text { Ferramenta } \\
\text { de agitação }\end{array}$ & $\begin{array}{c}\text { Velocidade } \\
\text { de agitação }\end{array}$ & $\begin{array}{c}\text { Tempo } \\
\text { de agitação }\end{array}$ \\
\hline 1 & -1 & -1 & -1 \\
\hline 2 & +1 & -1 & -1 \\
\hline 3 & -1 & +1 & -1 \\
\hline 4 & +1 & +1 & -1 \\
\hline 5 & -1 & -1 & +1 \\
\hline 6 & +1 & -1 & +1 \\
\hline 7 & -1 & +1 & +1 \\
\hline 8 & +1 & +1 & +1 \\
\hline
\end{tabular}


experimentais foi realizada utilizando o software Statistica, versão 5.0. Os valores codificados e reais das variáveis de entrada empregados no planejamento, encontram-se na Tabela I. $\mathrm{Na}$ Tabela II, está apresentada a matriz de planejamento experimental.

\section{Preparação das dispersões}

As dispersões de argilas bentoníticas em água deionizada na concentração de 4,86\% em massa (24,3 g de argila seca em $500 \mathrm{~mL}$ de água deionizada) foram preparadas em agitador Hamilton Beach, modelo N 5000 [14], utilizando as variáveis de entrada de acordo com o planejamento experimental (Tabela II). As ferramentas de agitação F1, palheta corrugada com 2,5 cm de diâmetro, e F2, em forma de borboleta com partes móveis com 3,5 cm de diâmetro, estão apresentadas na Fig. 1. As velocidades e os tempos de agitação estudados foram de 10.000 rpm e $17.000 \mathrm{rpm}$ e de $10 \mathrm{~min}$ e $20 \mathrm{~min}$, respectivamente.

As dispersões, após preparadas, permaneceram em repouso por um período de 24 h em câmara úmida com $100 \%$ de umidade relativa.
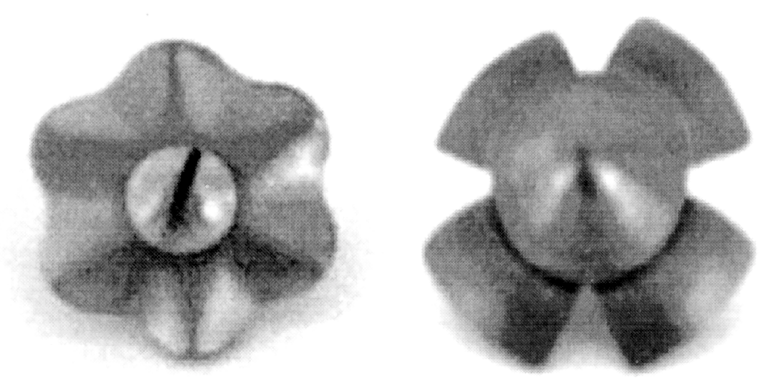

Figura 1: Ferramentas de agitação: F1 - ferramenta em forma de palheta corrugada com 2,5 cm de diâmetro e F2 - ferramenta em forma de borboleta com partes móveis com 3,5 cm de diâmetro.

[Figure 1: Agitation Tools: F1-2.5 cm diameter corrugated disk and $F 2-3.5 \mathrm{~cm}$ diameter butterfly form with four articulated parts.]

\section{Estudo reológico}

Foram determinadas as viscosidades aparente (VA) e plástica (VP) em viscosímetro Fann modelo 35A e o volume do filtrado (VF), em filtro prensa da marca Fann, segundo norma da Petrobras [14].

\section{RESULTADOS E DISCUSSÃO}

Nas Tabelas III, IV, V e VI, encontram-se os resultados de viscosidade aparente (VA), viscosidade plástica (VP) e volume de filtrado (VF) obtidos com as dispersões das amostras Dolomil, Fungel, Brasgel e Brasgel PA, respectivamente, preparadas de acordo com planejamento experimental contido na Tabela II.

Analisando os resultados de viscosidade aparente apresentados pelas dispersões das argilas estudadas, observou-se que o aumento no tempo e na velocidade de agitação conduziu as dispersões a maiores valores de VA. Este aumento deve-se, provavelmente, a maior hidratação e separação das partículas de argila, que resulta na diminuição da água livre e aumento na viscosidade aparente do sistema. Segundo Lummus e Azar [2], quando uma suspensão de argila é cisalhada ocorre a quebra de ligações químicas da sua estrutura, resultando na exposição de valências positivas dos átomos das folhas tetraédricas e octaédricas, negativas dos átomos de oxigênio, ou ambas, dependendo de onde ocorra a quebra. Estas cargas retêm íons que adsorvem água promovendo a formação de moléculas solvatadas de grandes dimensões e a diminuição da água livre disponível. Logo, o aumento na taxa de cisalhamento e no tempo de agitação, favoreceu a quebra destas ligações químicas, que em maior número, levou as dispersões estudadas a apresentarem

Tabela III - Viscosidades aparente (VA) e plástica (VP) e volume de filtrado (VF) das dispersões da amostra Dolomil.

[Table III - Apparent and plastic viscosity and fluid loss of Dolomil dispersions.]

\begin{tabular}{lccc}
\hline Experimentos & \multicolumn{3}{c}{ Propriedades reológicas } \\
\cline { 2 - 4 } & $\mathrm{VA}(\mathrm{cP})$ & $\mathrm{VP}(\mathrm{cP})$ & $\mathrm{VF}(\mathrm{mL})$ \\
\hline 1 (F1, 10.000 rpm e 10 min) & 8,0 & 4,0 & 21,5 \\
\hline 2 (F2, 10.000 rpm e 10 min) & 9,1 & 3,8 & 20,5 \\
\hline 3 (F1, 17.000 rpm e 10 min) & 9,8 & 4,3 & 20,5 \\
\hline 4 (F2, 17.000 rpm e 10 min) & 11,5 & 4,3 & 18,8 \\
\hline $5(\mathrm{~F} 1,10.000$ rpm e 20 min) & 9,2 & 4,0 & 21,0 \\
\hline 6 (F2, 10.000 rpm e 20 min) & 9,8 & 4,0 & 20,8 \\
\hline 7 (F1, 17.000 rpm e 20 min) & 11,8 & 4,3 & 20,5 \\
\hline 8 (F2, 17.000 rpm e 20 min) & 13,6 & 3,5 & 18,3 \\
\hline Valores especificados [9] & $\geq 15,0$ & $\geq 4,0$ & $\leq 18,0$ \\
\hline
\end{tabular}

Tabela IV - Viscosidades aparente (VA) e plástica (VP) e volume de filtrado (VF) das dispersões da amostra Fungel.

[Table IV - Apparent and plastic viscosity and fluid loss of Fungel dispersions.]

\begin{tabular}{lccc}
\hline Experimentos & \multicolumn{3}{c}{ Propriedades reológicas } \\
\cline { 2 - 4 } & $\mathrm{VA}(\mathrm{cP})$ & $\mathrm{VP}(\mathrm{cP})$ & $\mathrm{VF}(\mathrm{mL})$ \\
\hline 1 (F1, 10.000 rpm e 10 min) & 4,9 & 4,0 & 21,0 \\
\hline 2 (F2, 10.000 rpm e 10 min) & 4,8 & 3,3 & 19,7 \\
\hline 3 (F1, 17.000 rpm e 10 min) & 5,0 & 3,5 & 19,5 \\
\hline 4 (F2, 17.000 rpm e 10 min) & 6,1 & 4,0 & 19,5 \\
\hline 5 (F1, 10.000 rpm e 20 min) & 5,3 & 3,8 & 19,5 \\
\hline 6 (F2, 10.000 rpm e 20 min) & 6,1 & 3,8 & 20,7 \\
\hline 7 (F1, 17.000 rpm e 20 min) & 6,6 & 4,3 & 18,3 \\
\hline 8 (F2, 17.000 rpm e 20 min) & 6,6 & 4,0 & 17,0 \\
\hline Valores especificados [9] & $\geq 15,0$ & $\geq 4,0$ & $\leq 18,0$ \\
\hline
\end{tabular}


Tabela V - Viscosidades aparente (VA) e plástica (VP) e volume de filtrado (VF) das dispersões da amostra Brasgel.

[Table V-Apparent and plastic viscosity and fluid loss of Brasgel dispersions.]

\begin{tabular}{|c|c|c|c|}
\hline \multirow[t]{2}{*}{ Experimentos } & \multicolumn{3}{|c|}{ Propriedades reológicas } \\
\hline & $\mathrm{VA}(\mathrm{cP})$ & $\mathrm{VP}(\mathrm{cP})$ & $\mathrm{VF}(\mathrm{mL})$ \\
\hline $1(\mathrm{~F} 1,10.000 \mathrm{rpm}$ e $10 \mathrm{~min})$ & 5,6 & 4,5 & 20,5 \\
\hline $2(\mathrm{~F} 2,10.000 \mathrm{rpm}$ e $10 \mathrm{~min})$ & 5,6 & 4,0 & 20,3 \\
\hline 3 (F1, 17.000 rpm e $10 \mathrm{~min})$ & 6,0 & 4,5 & 19,8 \\
\hline $4(\mathrm{~F} 2,17.000 \mathrm{rpm}$ e $10 \mathrm{~min})$ & 7,0 & 4,5 & 17,7 \\
\hline 5 (F1, 10.000 rpm e $20 \mathrm{~min})$ & 6,4 & 4,3 & 19,5 \\
\hline $6(\mathrm{~F} 2,10.000 \mathrm{rpm}$ e $20 \mathrm{~min})$ & 6,9 & 4,3 & 20,5 \\
\hline 7 (F1, 17.000 rpm e $20 \mathrm{~min})$ & 8,3 & 5,0 & 19,8 \\
\hline $8(\mathrm{~F} 2,17.000 \mathrm{rpm}$ e $20 \mathrm{~min})$ & 8,4 & 4,3 & 17,3 \\
\hline Valores especificados [9] & $\geq 15,0$ & $\geq 4,0$ & $\leq 18,0$ \\
\hline
\end{tabular}

Tabela VI - Viscosidades aparente (VA) e plástica (VP) e volume de filtrado (VF) das dispersões da amostra Brasgel PA.

[Table VI - Apparent and plastic viscosity and fluid loss of Brasgel PA dispersions.]

\begin{tabular}{lccc}
\hline Experimentos & \multicolumn{3}{c}{ Propriedades reológicas } \\
\cline { 2 - 4 } & $\mathrm{VA}(\mathrm{cP})$ & $\mathrm{VP}(\mathrm{cP})$ & $\mathrm{VF}(\mathrm{mL})$ \\
\hline 1 (F1,10.000 rpm e 10min) & 11,5 & 4,3 & 18,3 \\
\hline 2 (F2,10.000 rpm e 10min) & 12,8 & 4,8 & 17,3 \\
\hline 3 (F1,17.000 rpm e 10min) & 12,9 & 4,8 & 17,5 \\
\hline $4(\mathrm{~F} 2,17.000$ rpm e 10min) & 14,0 & 4,0 & 16,3 \\
\hline 5 (F1,10.000 rpm e 20min) & 12,6 & 4,3 & 18,0 \\
\hline 6 (F2,10.000 rpm e 20min) & 15,5 & 4,0 & 18,0 \\
\hline $7(\mathrm{~F} 1,17.000$ rpm e 20min) & 16,1 & 4,0 & 16,3 \\
\hline 8 (F2, 17.000 rpm e 20min) & 15,3 & 4,3 & 16,3 \\
\hline Valores especificados [9] & $\geq 15,0$ & $\geq 4,0$ & $\leq 18,0$ \\
\hline
\end{tabular}

maior viscosidade aparente.

Observou-se também que as dispersões preparadas com a ferramenta F2 (em forma de borboleta) apresentaram pequeno aumento em VA; provavelmente, as partes móveis desta ferramenta proporcionaram uma maior dispersão das partículas de argila, facilitando o mecanismo de hidratação.

Embora a viscosidade plástica tenha se apresentado praticamente constante, não havendo variações significativas com o aumento da velocidade e/ou tempo de agitação, os maiores valores foram obtidos quando as dispersões foram preparadas com o uso da ferramenta F1, na velocidade de $17.000 \mathrm{rpm}$.

Com relação ao volume de filtrado, verificou-se, em média, que menores valores foram obtidos quando do uso da ferramenta F2 e da velocidade de agitação de $17.000 \mathrm{rpm}$, para qualquer tempo de agitação. Para as dispersões preparadas com as argilas Dolomil, Brasgel e Brasgel PA (Tabelas III, V e VI), observou-se uma relação entre a viscosidade aparente e o volume de filtrado; aumento da VA com queda em VF. Este comportamento é o esperado, uma vez que o volume de filtrado é uma medida da energia de ligação entre as partículas de argila e as moléculas de água. Portanto, se esta energia de ligação é alta, a viscosidade aparente é elevada e o volume de filtrado é baixo, ou seja, o sistema apresenta pequena quantidade de água livre.

Comparando os resultados dos parâmetros reológicos das dispersões estudadas (Tabelas III, IV, V e VI) com as especificações da Petrobras [9] para uso como fluidos de perfuração de poços de petróleo de base água, observou-se que apenas as dispersões preparadas com a argila Brasgel PA apresentaram médias dentro dos limites propostos.

Na Tabela VII, estão apresentados os resultados da análise estatística (\% de variação explicada) das propriedades reológicas das dispersões preparadas com as amostras Dolomil, Fungel, Brasgel e Brasgel PA.

Tabela VII - Análise estatística das propriedades reológicas das dispersões preparadas com as amostras Dolomil, Fungel, Brasgel e Brasgel PA.

[Table VII - Statistical analyze of rheological properties of Dolomil, Fungel, Brasgel and Brasgel PA dispersions.]

\begin{tabular}{llll}
\hline \multirow{2}{*}{ Amostras } & \multicolumn{3}{c}{ \% de variação explicada* } \\
\cline { 2 - 4 } & $\mathrm{VA}$ & $\mathrm{VP}$ & $\mathrm{VF}$ \\
\hline Dolomil & 99,8 & 77,5 & 97,0 \\
\hline Fungel & 87,6 & 59,8 & 84,0 \\
\hline Brasgel & 97,0 & 68,7 & 97,0 \\
\hline * $\mathrm{R}^{2}=\left(\mathrm{S}_{\mathrm{OR}} / \mathrm{S}_{\mathrm{QR}}\right) \times 100, \mathrm{~S}_{\mathrm{QR}}:$ soma quadrática da regressão e $\mathrm{S}_{\mathrm{Qr}}$ : soma \\
quadrática do resíduo
\end{tabular}

A análise de significância estatística (Tabela VII) mostrou que o \% de variação explicada dos resultados experimentais para VA e para VF foram satisfatórios, para as dispersões preparadas com as argilas estudadas. Para a VP, os valores obtidos foram baixos. É importante ressaltar que estes resultados são significativos para o nível de $95,0 \%$ de confiança.

Os modelos matemáticos codificados para as propriedades reológicas das dispersões das argilas estudadas estão apresentados na Tabela VIII.

As equações apresentadas na Tabela VIII, mostraram que as propriedades reológicas das dispersões estudadas não são influenciadas pelas variáveis de entrada, ou seja, o tipo de ferramenta, a velocidade e o tempo de agitação não interferem de forma estatisticamente significativa nas viscosidades aparente e plástica e no volume de filtrado das dispersões das argilas bentoníticas estudadas, ao nível de $95,0 \%$ de confiança. Em outras palavras, o comportamento reológico das dispersões das argilas não é significativamente afetado quando da substituição da ferramenta F1 pela F2, do aumento da velocidade de $10.000 \mathrm{rpm}$ para $17.000 \mathrm{rpm}$ e do aumento do tempo de agitação de 10 para 20 minutos. 
Tabela VIII - Modelos matemáticos codificados para as propriedades reológicas das dispersões das argilas Dolomil, Fungel, Brasgel e Brasgel PA.

[Table VIII - Mathematics models of rheological properties of Dolomil, Fungel, Brasgel e Brasgel PA dispersions.]

Dispersões preparadas com a argila Dolomil

$\mathrm{VA}(\mathrm{cP})=10,35^{*}+0,65 \mathrm{~F}+1,33 \mathrm{v}+0,75 \mathrm{t}+0,22 \mathrm{Fv}-0,05 \mathrm{Ft}+0,28 \mathrm{vt}$

$\mathrm{VP}(\mathrm{cP})=4,03 *-0,12 \mathrm{~F}+0,07 \mathrm{v}-0,07 \mathrm{t}-0,07 \mathrm{Fv}-0,07 \mathrm{Ft}-0,12 \mathrm{vt}$

$\mathrm{VF}(\mathrm{mL})=20,24 *-0,64 \mathrm{~F}-0,71 \mathrm{v}-0,08 \mathrm{t}-0,03 \mathrm{Fv}-0,03 \mathrm{Ft}-0,03 \mathrm{vt}$

Dispersões preparadas com a argila Fungel

$\mathrm{VA}(\mathrm{cP})=5,68 *+0,45 \mathrm{~F}+0,80 \mathrm{v}+0,95 \mathrm{t}+0,10 \mathrm{Fv}-0,05 \mathrm{Ft}+0,10 \mathrm{vt}$

$\mathrm{VP}(\mathrm{cP})=3,84 *-0,12 \mathrm{~F}+0,22 \mathrm{v}+0,28 \mathrm{t}+0,22 \mathrm{Fv}-0,02 \mathrm{Ft}+0,12 \mathrm{vt}$

$\mathrm{VF}(\mathrm{mL})=19,40^{*}-0,35 \mathrm{~F}-1,65 \mathrm{v}-1,05 \mathrm{t}-0,30 \mathrm{Fv}+0,30 \mathrm{Ft}-0,80 \mathrm{vt}$

Dispersões preparadas com a argila Brasgel

\begin{tabular}{l}
$\mathrm{VA}(\mathrm{cP})=6,78^{*}+0,20 \mathrm{~F}+0,65 \mathrm{v}+0,73 \mathrm{t}+0,07 \mathrm{Fv}-0,05 \mathrm{Ft}+0,20 \mathrm{vt}$ \\
\hline $\mathrm{VP}(\mathrm{cP})=4,43-0,15 \mathrm{~F}+0,15 \mathrm{v}+0,05 \mathrm{t}-0,02 \mathrm{Fv}-0,02 \mathrm{Ft}+0,02 \mathrm{vt}$ \\
$\mathrm{VF}(\mathrm{mL})=19,43^{*}-0,48 \mathrm{~F}-0,78 \mathrm{v}-0,15 \mathrm{t}-0,68 \mathrm{Fv}+0,10 \mathrm{Ft}+0,05 \mathrm{vt}$
\end{tabular}

Dispersões preparadas com a argila Brasgel PA

$\mathrm{VA}(\mathrm{cP})=13,84^{*}+1,12 \mathrm{~F}+1,47 \mathrm{v}+2,07 \mathrm{t}-0,97 \mathrm{Fv}-0,07 \mathrm{Ft}+0,17 \mathrm{vt}$

$\mathrm{VP}(\mathrm{cP})=4,31 *-0,07 \mathrm{~F}-0,07 \mathrm{v}-0,32 \mathrm{t}-0,17 \mathrm{Fv}+0,07 \mathrm{Ft}+0,07 \mathrm{vt}$

$\mathrm{VF}(\mathrm{mL})=17,25^{*}+0,55 \mathrm{~F}-1,30 \mathrm{v}-0,20 \mathrm{t}-0,05 \mathrm{Fv}+0,55 \mathrm{Ft}-0,40 \mathrm{vt}$

Sendo: F a ferramenta, v a velocidade e to tempo de agitação.

* Estatisticamente significativos ao nível de 95,0\% de confiança.

Através deste estudo, observou-se que o tipo de ferramenta utilizado no preparo de dispersões de argilas bentoníticas (ferramenta em forma de palheta com ressaltos e em forma de borboleta com partes móveis), não compromete o seu comportamento reológico. Contudo, em trabalho anterior, Gomes e colaboradores [11], mostraram que o uso de ferramentas de agitação do tipo rotor-estator, que operam com elevadas tensões de cisalhamento, provocam cominuição das partículas de argila com conseqüente elevação da sua área específica, assim como maiores interações elétricas entre o sistema disperso, meio dispersor e as partículas solvatadas, tendo como resultado reologias menos eficientes.

\section{CONCLUSÕES}

Com o objetivo de estudar a influência do tipo de ferramenta, da velocidade e do tempo de agitação nas propriedades reológicas de dispersões de quatro amostras de argilas bentoníticas sódicas industrializadas no Estado da Paraíba,
Brasil, concluiu-se que:

-as variáveis de entrada (tipo de ferramenta, a velocidade e o tempo de agitação) não influenciam estatisticamente, ao nível de $95,0 \%$ de confiança, o comportamento reológico das dispersões de argilas bentoníticas e

-apenas as dispersões preparadas com a argila Brasgel PA apresentam-se dentro dos padrões especificados pela Petrobras para uso como fluidos de perfuração de poços de petróleo.

\section{AGRADECIMENTOS}

Os autores agradecem à Agência Nacional do Petróleo ANP e ao CNPq/CTPETRO pelo apoio financeiro. L. V. Amorim é aluna de Doutorado em Eng. de Processos, Bolsista ANP/ PRH-25. C. M. Gomes é aluna de Graduação em Eng. de Materiais, Bolsista ANP/PRH-25.

\section{REFERÊNCIAS}

[1] M. H. A. Trindade, "Bentonita”, www.dnpm.org.br, 20 de setembro (2001).

[2] J. L. Lummus, J. J. Azar, "Drilling fluids optimization: a practical field approach", PennWell Publishing Company, Tulsa, Oklahoma (1986) 19, 101.

[3] R. K. Clark, J. Petrol. Technol. 46, 9 (1994) 804-809.

[4] R. Caenn, G. V. Chillingar, J. Petrol. Sci. Eng. 14, 3-4 (1996) 221-230.

[5] H. C. H. Darley, G. R. Gray, "Composition and properties of drilling and completion fluids", Fifth Edition, Gulf Publishing Company, Houston, Texas (1988) 2.

[6] P. Souza Santos, "Ciência e tecnologia de argilas", Editora Edgard Blücher, S. Paulo, Brasil (1989) 2, 609.

[7] Petrobrás, Argila Ativada para Água Doce, EE-78, (1968).

[8] P. Stefan, Cerâmica 12, 47-48 (1966) 266.

[9] Petrobrás, "Viscosificante para fluido de perfuração base de água na exploração e produção de petróleo", Especificação N2604 (1998).

[10] L. M. C. Assunção, H. C. Ferreira, Cerâmica 25, 114 (1979) 165. [11] C. M. Gomes, L. V. Amorim, H. C. Ferreira, in Proceedings Jornadas SAM-CONAMET-ASS, Posadas, Argentina, setembro (2001) no prelo.

[12] L. V. Amorim, "Otimização da reologia e da durabilidade de fluidos de perfuração à base de água e argila", Exame de Qualificação, Doutorado em Eng. de Processos, Universidade Federal da Paraíba, (2001).

[13] B. Barros Neto, I. S. Scarminio, R. E. Bruns, "Planejamento e otimização de experimentos", Editora da UNICAMP, S. Paulo, Brasil (1996) 79.

[14] Petrobrás, "Ensaio de viscosificante para fluido de perfuração base de água na exploração e produção de petróleo", Método N-2605 (1998).

(Rec. 09/04/02, Ac. 21/09/02) 